\title{
IGHV4-34 Positive
}

National Cancer Institute

\section{Source}

National Cancer Institute. IGHV4-34 Positive. NCI Thesaurus. Code C133813.

An indication that IGHV4-34 expression has been detected in a sample. 